\title{
Desarrollo gradual e integral
}

\author{
ÁNGEL GALINDO GARCÍA* \\ Universidad Pontificia de Salamanca (España) \\ agalindo@upsa.es
}

\begin{abstract}
Resumen
$\mathrm{El}$ autor distribuye este trabajo en los siguientes apartados: plantea la diferencia entre el concepto de desarrollo y de progreso, consciente de que después de estos cincuenta años, con la evolución tanto de la economía como de las ideologías, y después de una gran crisis social y financiera, es necesario distinguir conceptualmente y en la praxis estos dos términos; situamos la reflexión en el horizonte de la agenda 2030. Desde esta perspectiva estudia la propuesta de PP sobre el desarrollo y la evolución y dinamismo de este pensamiento a través de las encíclicas sociales posteriores hasta nuestros días dedicando un apartado especial a su relación con la ecología integral. Finalmente, a modo de conclusión, presenta los principios que fundamentan la gradualidad del desarrollo.
\end{abstract}

Palabras clave: desarrollo, dinamismo, gradual, agenda, solidaridad, sostenibilidad.

\section{Gradual and integral development}

\begin{abstract}
The author distributes this work in the following sections: he points out the difference between the concept of development and progress, aware that after these fifty years, with the evolution of both economy and ideologies, and after a great social crisis and financial, it is necessary to distinguish conceptually and praxis these two terms; we place the reflection on the horizon of the 2030 agenda. From this perspective, he studies the proposal of Populorum progressio on the development and evolution and dynamism of this thought through the social encyclicals that have been up until now, devoting a special section to its relation with integral ecology. Finally, by way of conclusion, it presents the principles underlying the gradual development.
\end{abstract}

Key words: development, dynamism, gradual, agenda, solidarity, sustainability.

\footnotetext{
* Sacerdote y Catedrático de Teología Moral de la Facultad de Teología de la UPSA. Ha sido Decano de la misma Facultad y Rector de la Universidad, así como Director del Instituto Superior de Ciencias de la Familia (UPSA) y es director de la revista de Caritas Española Corintios XIII. Entre sus abundantes publicaciones destacan los libros Moral socioeconómica (1996), Voluntariado y sociedad participativa (2004), Responsabilidad social corporativa y medios de comunicación social (2008), Caridad en la verdad. Comentario a la encíclica "Caritas in veritate" de Benedicto XVI (2010).

Propongo el siguiente artículo resultado del trabajo realizado durante mi estancia en el centro de la Iglesia Nacional Española de Santiago y Monserrat de Roma durante el mes de marzo de 2017.
} 


\section{INTRODUCCIÓN}

Hace diez años, Benedicto XVI reflexionó sobre la actualidad de Populorum progressio en la encíclica Caritas in veritate: "A más de cuarenta años de su publicación, la relectura de la Populorum progressio insta a permanecer fieles a su mensaje de caridad y de verdad, considerándolo en el ámbito del magisterio específico de Pablo VI y, más en general, dentro de la tradición de la doctrina social de la Iglesia" (CV, 10). Años antes, había hecho lo mismo Juan Pablo II (1987) en Sollicitudo rei socialis.

La revista Veritas del Pontificio Seminario Mayor "San Rafael" de Valparaíso (Chile), tiene como proyecto dedicar un número a estudiar "La dinámica del desarrollo: ética y doctrina social de la Iglesia" con motivo del 50 aniversario de la publicación de Populorum progressio. Agradezco a los organizadores la oportunidad que me ofrecen para investigar una vez más sobre el tema del desarrollo, cuando el papa Francisco promueve esta misma efeméride a través del nuevo Consistorio ${ }^{1}$.

El tema es actual y están apareciendo numerosas colaboraciones con esta efeméride. Con la aparición de la encíclica Caritas in veritate del papa Benedicto XVI en el 2009 con motivo del cuarenta aniversario de PP tuve la oportunidad de acercarme al tema del desarrollo. Anteriormente, en relación con la encíclica de san Juan Pablo II Solicitudo rei socialis, también fue una ocasión, de acuerdo a mí especialidad, para reflexionar sobre la ética del desarrollo. En ambos casos son varios los trabajos de investigación que hemos podido ofrecer (Galindo García, 2005; 1988).

Después de la publicación de los trabajos del papa Francisco, Laudato si' y Evangelii gaudium, la perspectiva se sitúa en el marco del desarrollo sostenible en el contexto de la preparación de la agenda 2030 de Naciones unidas y ante el problema causado con la crisis y con las cuestiones ecológicas.

Dividiré este trabajo en los siguientes apartados: después de aclarar la diferencia entre desarrollo y progreso, situamos la reflexión en el horizonte de la agenda 2030. Desde esta perspectiva estudiamos la propuesta de PP sobre el desarrollo y la evolución de este pensamiento a través de las encíclicas sociales posteriores hasta nuestros días y en relación con la ecología integral. Finalmente, a modo de conclusión, presentaremos los principios que fundamentan la gradualidad del desarrollo.

\footnotetext{
1 El Papa Francisco, de conformidad con el motu proprio "Progressionem Humanam" del 17 de agosto de 2016, instituyó el Dicasterio para la Promoción del Desarrollo Humano Integral.
} 


\section{1. ¿DESARROLLO O PROGRESO? ${ }^{2}$}

Después de estos cincuenta años, con la evolución tanto de la economía como de las ideologías, y después de una gran crisis social y financiera, es necesario distinguir conceptualmente y en la praxis los términos desarrollo y progreso. Bajo la denominación de "progreso" o "progresistas" se sitúan una pléyade de personas que, aparentando ser avanzados en la práctica, se manifiestan con gestos tradicionales y retrógrados ${ }^{3}$.

Por otro lado, la sociología nos dice que progresista es aquel que trabaja por el desarrollo de las naciones más que el de las personas o ciudadanos de dicho país. Sin embargo, el "desarrollista" es el que busca el desarrollo integral de todas y cada una de las personas o habitantes de un país. En el primer caso se promueve una economía competitiva y en el segundo una economía humanística.

Asimismo, el término 'desarrollo' tiene connotaciones futuristas de forma que el auténtico desarrollo cae bajo la actitud de aquellos que miran hacia el futuro en sus actuaciones y propuestas para conseguir unos fines que les perfeccionan. En este carácter futurista y en evolución situamos esta reflexión sobre el dinamismo del desarrollo.

Las palabras ‘progreso’ y 'desarrollo' aparecen en la versión latina de la encíclica Populorum progressio como progressio. La encíclica Sollicitudo rei socialis (SRS) no conoce la diferencia en latín. Califica el término progressio cada vez como positivo o negativo. De todos modos, el motivo inspirador es el 'desarrollo de los pueblos' (SRS, 4).

En su carta apostólica Octogessima adveniens el beato Pablo VI describe la ambigüedad del progreso, en cuanto para un cristiano el progreso encuentra necesariamente el misterio escatológico de la muerte: "Difundida por los medios modernos de información y por el estímulo del saber y la generalización del afán de consumo, el progreso se convierte en ideología omnipresente. Por tanto, se plantea hoy la duda sobre su valor y sobre su origen. Para cristianos y cristianas, el progreso encuentra necesariamente el misterio escatológico de la muerte; la muerte de Cristo y su resurrección, así como el impulso del Espíritu del Señor, ayudan a la persona a situar su libertad creadora y agradecida en la verdad de cualquier progreso y en la única esperanza que no decepciona jamás" (OA, 41).

2 Conferencia impartida por el profesor Fabrice Hadjadj sobre Progreso: ¿el mito del desarrollo?, el día 4 de abril de 2017 en el Congreso sobre los 50 años de la Populorum progressio. Véase Gamband (2012: 80); Longchamp (2008).

Puede verse como John Gray ha estudiado a Isaiah Berlin, la relación entre política y religión - de Al Qaeda al neoconservadurismo estadounidense-, la noción de "progreso" en la modernidad y las visiones del ecologismo (Gray, 2011). 
Por otra parte, el papa Francisco argumenta en Laudato si' que la educación medioambiental debe incluir la crítica del mito moderno del 'progreso' (LS, 143). Como vemos en la crítica de Octogessima adveniens al progreso recurre a la imagen de una marcha, de un lugar a otro y de una ruptura. El desarrollo inspira más bien la idea de un crecimiento orgánico, de algo ya presente que es en germen de manera armoniosa. De este modo el progreso que mantiene el sujeto aparece más conservador que el desarrollo que implica un desarrollo del hombre en su integridad. Para los progresistas hoy en día el progreso tecnológico parece un verdadero avance, pero no se trata de un progreso técnico. De hecho, el progreso técnico es una regresión técnica ya que se saben hacer cada vez menos cosas [LS, 105; CV, capítulo VI] (Guardini, 1958).

El progreso implica la existencia de un objetivo. Sin objetivo claro, ¿cómo medir el progreso? Sin embargo, para los progresistas el progreso es inevitable y es imposible volver atrás, pero entonces ¿dónde está la libertad? Hoy nos damos cuenta de la abdicación de la política ante el progreso tecnológico. En este caso, la democracia se convierte en una ilusión.

Sollicitudo rei socialis 28 describe una especie de sobre-desarrollo que es desproporcionado y que crea una cultura de desecho con una aceleración de la caducidad: "Debería ser altamente instructiva una constatación desconcertante de este período más reciente: junto a las miserias del subdesarrollo, que son intolerables, nos encontramos con una especie de superdesarrollo, igualmente inaceptable porque, como el primero, es contrario al bien y a la felicidad auténtica. En efecto, este superdesarrollo, consistente en la excesiva disponibilidad de toda clase de bienes materiales para algunas categorías sociales, fácilmente hace a los hombres esclavos de la 'posesión' y del goce inmediato, sin otro horizonte que la multiplicación o la continua sustitución de los objetos que se poseen por otros todavía más perfectos. Es la llamada civilización del 'consumo' o consumismo, que comporta tantos 'desechos' o 'basuras'. Un objeto poseído, y ya superado por otro más perfecto, es descartado simplemente, sin tener en cuenta su posible valor permanente para uno mismo o para otro ser humano más pobre".

La llamada de Pablo VI del desarrollo de cada hombre y de todos los hombres es una especie de eco del grito de Albert Camus en su discurso Nobel para evitar que el mundo se deshaga. Así, el mayor progreso hoy es ser conservador. La propuesta de la abolición del hombre sale de buenas intenciones, pero reconozcamos la permanencia del mal en el mundo sin dejar de querer hacer el bien y recordemos el misterio cristiano: la cruz participa en la gloria y el martirio no es un contratiempo ${ }^{4}$.

4 Véase F. Hadjadj, Progreso: ¿el mito del desarrollo?, conferencia impartida en la jornada organizada por el Consistorio sobre "a los 50 años de PP". 
Las diversas conferencias mundiales sobre la ecología y la contaminación han puesto en evidencia la importancia del desarrollo y la invitación a una más justa distribución de los bienes. Por otra parte, la crisis financiera iniciada en 2007 ha hecho que naciones unidas repensara las estrategias mundiales del desarrollo. En este contexto y como respuesta a los objetivos del milenio ha nacido la propuesta de la agenda 2030.

El desarrollo sustentable se ha transformado en el paradigma de esta época. Pero los problemas que sufre el ecosistema del planeta no se resuelven con hacer sustentable el desarrollo. La superpoblación, el consumo desmedido y dilapidador y una economía que no considera los costos ambientales, son factores que harán inhabitable el planeta en poco tiempo. La agenda 2030 es una invitación para abrir el debate sobre las verdaderas causas de los desequilibrios que son cada día más notorios y de los peligros a los que nos enfrentamos agotando sin piedad los recursos naturales disponibles.

La especialista Doña Mercedes Ruiz-Giménez, integrante de la Delegación oficial de España en la 70 Asamblea General de Naciones Unidas, en su calidad de presidenta de la Coordinadora de ONG para el Desarrollo reflexiona sobre lo acontecido, y analiza los retos a los que se enfrenta la humanidad en los próximos años para alcanzar la llamada Agenda 2030. Sugiere un abordaje integral, la realización de planes nacionales, así como políticas internacionales orientados a cumplir los objetivos de Desarrollo Sostenible.

La $70^{a}$ Asamblea General de Naciones Unidas no fue una asamblea más; el encuentro dio el pistoletazo de salida a una nueva agenda internacional para luchar contra la pobreza y las desigualdades y para proteger el medioambiente. Los compromisos asumidos por 190 jefes de Estado y de gobierno marcarán sus responsabilidades para los próximos 15 años. Ahora se trata de ver cómo empieza este camino y, sobre todo, a dónde va a llevarnos.

La senda que comienza viene marcada por luces y sombras. La brújula la pondrán los Objetivos de Desarrollo Sostenible, 17 objetivos que contemplan temas como la educación, la salud, el cambio climático, el hambre, la igualdad de género, la paz o el derecho al agua, entre otros. La amplitud es enorme, como enormes son los retos que afrontamos como humanidad. El abordaje integral de todos ellos será clave en un mundo tan interconectado como el actual(LS'105-108).

La Agenda 2030 incorpora novedades relevantes respecto a propuestas anteriores que pueden suponer una oportunidad para el proceso que ahora se inicia. Por primera vez, una hoja de ruta de este tipo contempla 
que la lucha contra la pobreza, el desarrollo humano y el medioambiente van de la mano; aunque parece algo evidente, hasta ahora no se había contemplado de tal manera. El segundo elemento clave es el reconocimiento de que la desigualdad entre países, y en el interior de ellos, es un problema central que hay que abordar de manera integral y urgente. Además, se trata de una agenda universal que afecta a todos los países; es decir, que cada país deberá implementar un plan de acción para alcanzar los objetivos no solo en materia de cooperación internacional sino también en relación a las políticas públicas domésticas.

Hay ciertas cuestiones que han quedado en el aire y que pueden significar un freno importante para la consecución de los objetivos acordados. La primera de ellas es que el cumplimiento de estos objetivos es voluntario; y en relación con los compromisos internacionales, se ha demostrado que la buena fe no es suficiente. Por otra parte, se reitera el mantra del crecimiento económico como solución a nuestros problemas, ignorando que el crecimiento por sí solo no conduce al bienestar y, lo que es más grave, que vivimos en un planeta cuyos recursos naturales están al límite de su explotación —este tema ha sido denunciado por el propio Papa Francisco en la sede de la ONU-. Otro elemento preocupante es cómo los derechos humanos han ido perdiendo fuerza a lo largo de las negociaciones. Por un lado, han desaparecido en partes fundamentales del texto y, por otro, cuando se habla del acceso a la educación, sanidad o alimentación se evita mencionar que estamos ante derechos humanos fundamentales.

La puesta en marcha de esta hoja de ruta exigiría, en primer lugar, cambios profundos en el sistema económico y político internacional, y eso, de momento, no parece que se vaya a dar. Si no atajamos las causas, nunca acabaremos con sus consecuencias. Seguir como hasta ahora no es una opción.

Es el momento de pasar de las palabras a los hechos. Y eso pasa, ineludiblemente, por la creación de un Plan Nacional de Implementación en cada país que sea definido con la participación de los distintos sectores implicados: academia, sociedad civil, parlamento, sindicatos... Y que además, contemple todas las políticas públicas que deben contribuir a la lucha contra la pobreza y la desigualdad, tanto dentro como fuera de nuestras fronteras. Es la hora de la llamada coherencia de políticas; es decir, de garantizar que nuestras políticas económicas, comerciales, energéticas, migratorias... son coherentes con la defensa de los derechos humanos aquí y en cualquier lugar del mundo.

Llevar a cabo una agenda tan ambiciosa como esta exige una financiación suficiente; sin los medios necesarios es imposible que los compromisos se hagan realidad. Es preocupante que ésta sea una de sus grandes debilidades. Los gobiernos no parecen estar dispuestos a hacer grandes 
cambios en las reglas del juego y, sin cambios a fondo en el status quo, la agenda difícilmente será transformadora. La financiación pública es la única que puede garantizar la dotación necesaria para implementar las políticas adecuadas.

La puesta en marcha de la agenda debe ir acompañada de políticas contra la evasión y los paraísos fiscales. No se puede exigir responsabilidad a los países empobrecidos a través de lo que se denomina "movilización doméstica de recursos", sin que se establezcan medidas contra la evasión y los paraísos fiscales. Además, debe establecerse un mecanismo independiente que aborde los problemas de endeudamiento insostenible de los Estados - un serio problema que afecta tanto a los países empobrecidos, como a los países europeos, tal como la crisis griega ha puesto de manifiesto-; sin ese mecanismo los países con más poder podrán seguir imponiendo sus condiciones al resto.

Nunca antes el mundo estuvo tan interconectado como ahora. El abordaje de los problemas que nos afectan como sociedad no puede ser sino global; afrontarlos desde una óptica local o nacional nos llevaría a un callejón sin salida en el que se alcanzarían parches y no soluciones reales.

Desde la sociedad civil estaremos muy pendientes de las decisiones que se vayan tomando (o no) para garantizar que las propuestas de esta agenda - llamada Agenda 2030 - se aplican, tanto en las políticas nacionales como en las internacionales. Confiamos en que se habiliten espacios para que esa participación en los asuntos públicos sea asegurada. Se trata de garantizar que en el centro de las políticas se encuentran los derechos de las personas y la protección del planeta en el que vivimos ${ }^{5}$.

3. ANTE LA PROPUESTA DE PP SOBRE DESARROLLO: DERECHO Y FUNDAMENTO

La DSI, especialmente los documentos de Pablo VI, de Juan Pablo II y de los dos últimos pontífices, ha señalado insistentemente causas que provocan la distancia Norte y Sur o, con el lenguaje del papa Francisco, la sociedad del descarte y consecuentemente el subdesarrollo en los países pobres. Según estos pontífices, el subdesarrollo parece una situación imposible de solucionar si se piensa que solo se debe a las elecciones humanas equivocadas y se olvidan las razones que son resultado de mecanismos económicos, financieros y políticos (SRS, 16) y de estructuras de pecado (SRS, 36-39; LS, 107). Por ello, es necesario, en orden a descubrir la evolución del tratamiento que la Doctrina Social de la Iglesia ha hecho sobre

5 Mercedes Ruiz-Giménez, Presidenta de la Coordinadora de ONG para el Desarrollo, artículo publicado en el periódico público el 29 de septiembre de 2015. 
el progreso y desarrollo, constatar el derecho al desarrollo y su fundamento.

La encíclica Sollicitudo rei socialis insiste con fuerza en ello en un momento histórico de crisis radical y universal y señala, como causas del subdesarrollo, cuando el hombre vive la tensión entre el deseo de cultivar un desarrollo humano pleno y la realidad de unas estructuras de pecado destructoras de este deseo. "Es, pues, necesario individuar las causas de orden moral que, en el plano de la conducta de los hombres, considerados como personas responsables, ponen un freno al desarrollo e impiden su realización plena. Igualmente, cuando se disponga de recursos científicos y técnicos, que mediante las necesarias y concretas decisiones políticas deben contribuir a encaminar finalmente los pueblos hacia un verdadero desarrollo, la superación de los obstáculos mayores sólo se obtendrá gracias a decisiones esencialmente morales, las cuales, para los creyentes y especialmente los cristianos, se inspirarán en los principios de la fe, con la ayuda de la gracia divina" (SRS, $35^{6}$ ).

Asimismo, en relación con el cuidado de la naturaleza y en fidelidad al principio del destino universal de los bienes y a su justa distribución, la DSI recuerda que los bienes naturales son limitados, aunque aún se desconozca el precipicio de esas limitaciones al ignorar las dimensiones de las capacidades humanas. Hay límites políticos manifestados en la incapacidad de algunos poderes para dar una solución al problema del desarrollo en la tensión Norte-Sur y Este-Oeste. Hay límites económicos cuyas manifestaciones más concretas son el subempleo, la deuda externa, la existencia de un cuarto mundo. Pero son aún más graves los límites existentes en las actitudes del hombre convertidos en pecados estructurales. Hay una tendencia a reducir al hombre a un instrumento o factor productivo y consumista (LS, 193; Benedicto XVI, Mensaje para la Jornada Mundial de la Paz. 2010, 9).

La actitud ética ante estas limitaciones, causa de las dificultades de algunos países para acceder al desarrollo, aparece recogida en un texto, resumen de otros, de Juan Pablo II: "Respecto al contenido y a los temas, cabe subrayar la conciencia que tiene la Iglesia, experta en humanidad, de escrutar los signos de los tiempos y de interpretarlos a la luz del evangelio; la conciencia, igualmente profunda de su misión de servicio, distinta de la función del Estado, aun cuando se preocupa de la suerte de las personas en concreto; la referencia a las diferencias clamorosas en la situación de estas mismas personas; la confirmación de la enseñanza conciliar, eco fiel de la secular tradición de la Iglesia, respecto al destino universal de los

$6 \quad$ Las fuentes de este texto son numerosas: PP, 44 veces; GS, 20 veces; RN, 2 veces; QA, 1 vez; MM, 3 veces; OA, 6 veces; LE, 3 veces; RH, 2 veces. 
bienes; el aprecio por la cultura y la civilización técnica que contribuyen a la liberación del hombre, sin dejar de reconocer sus límites y, finalmente, sobre el tema del desarrollo, propio de la encíclica, la insistencia sobre el deber gravísimo que atañe a las naciones más desarrolladas. El mismo concepto de desarrollo propuesto por la encíclica surge directamente de la importación que la Constitución pastoral da a este problema” (SRS, $7^{7}$ ).

También las crisis de las democracias exigen un planteamiento de la cuestión del subdesarrollo, ya que durante las últimas décadas, la voluntad política, los esfuerzos entre los bloques y las acciones políticas han sido insuficientes. En este mundo, dividido en bloques, abundan las ideologías rígidas, domina el imperialismo de nuevo cuño y las estructuras de pecado se han generalizado. Por ello, es necesario buscar los caminos del auténtico desarrollo frente a las contradicciones estructurales amparadas bajo una democracia formal.

Pero la crisis de las democracias se manifiesta especialmente en las contradicciones de sus mismas estructuras. Las estructuras de pecado, cuyo origen está en los pecados personales, son, a la vez, fuentes de otros pecados. Las manifestaciones más importantes de estas contradicciones son: estrechez de miras, cálculos políticos errados, decisiones económicas imprudentes. Las consecuencias son graves: deuda externa, mala distribución de los préstamos y nacimiento de un cuarto mundo.

Otra de las causas del subdesarrollo, señaladas por la DSI y recogidas en CDSI, es el fenómeno del consumismo. Está surgiendo una concepción global de la vida que puede llamarse "el consumismo". Éste consiste en el descubrimiento y fomento de nuevas necesidades y nuevas modalidades para su satisfacción. La simbiosis entre sociedad de consumo y Estado asistencial crea estructuras de pecado en cuanto impiden la plena realización de los que son oprimidos de diversas formas (Zampetti, 1991: 175; Flecha Andrés, 1990: 21-53). Porque, dice Juan Pablo II, cuando "se absolutiza la producción y el consumo de las mercancías y estas ocupan el centro de la vida social y se convierten en el único valor de la sociedad, no subordinado a ningún otro, la causa hay que buscarla no sólo y no tanto en el sistema económico mismo, cuanto en el hecho de que todo el sistema sociocultural, al ignorar la dimensión ética y religiosa, se ha debilitado, limitándose únicamente a la producción de bienes y servicios" (CA, 39). Como afirma el papa Francisco, la solución llegará por caminos diversos a los de la inequidad: "Los planes asistenciales, que atienden ciertas urgencias, sólo deberían pensarse como respuestas pasajeras. Mientras no se re-

Este texto está construido desde GS, 3, 4, 19, 57, 63-69, 86; PP, 9, 13, 14-21, 22, 41, 
suelvan radicalmente los problemas de los pobres, renunciando a la autonomía absoluta de los mercados y de la especulación financiera y atacando las causas estructurales de la inequidad, no se resolverán los problemas del mundo y en definitiva ningún problema. La inequidad es raíz de los males sociales" (EG, 202).

\subsection{Derecho al desarrollo (Segovia Bernabé, 2008)}

La colaboración y ayuda a los países pobres, recuerda el CDSI, es un deber que nace del derecho que todos los pueblos tienen a un desarrollo integral. El desarrollo no es solo una aspiración sino también un derecho y como consecuencia una obligación ${ }^{8}$. Por ello las dificultades deben ser afrontadas con determinación firme y perseverante (SRS, 32).

Una forma particular de cooperación internacional es "al desarrollo". Esta es considerada como la finalidad y el motivo principal que se ha de tener presente en cualquier acción que quiera eliminar los obstáculos que hacen que muchos pueblos tengan un desarrollo precario. Las denuncias de esta situación por parte del Magisterio han sido continuas (CA, 33, 35). Con el fin de presentar una breve radiografía de esta cooperación para el desarrollo entresacamos del CDSI algunas indicaciones:

En primer lugar, puede constatarse en la sociedad moderna, quebrada por muchas desigualdades, que dentro del derecho de los hombres a participar activamente en la vida pública, sus posibilidades son mínimas. $\mathrm{Ha}$ de aceptarse el que los pueblos del tercer mundo tienen derecho a participar en las decisiones políticas de sus respectivos países, ya que el hombre debe ser siempre el protagonista de su propio desarrollo y destino (SRS, 30).

Asimismo, la evolución de la vida pública, no debe dejarse sólo en manos del Estado ni al servicio del mismo, sino a iniciativa de la sociedad y al servicio del hombre (Maritain, 1983). Este derecho a construir el propio destino ha de ser pleno, es decir, ha de extenderse a lo económico y social, al mundo cultural y espiritual, aunque el camino de este desarrollo auténtico sea largo y complejo, dada la intrínseca fragilidad del hombre y la situación precaria originada por el carácter mutable de la vida humana (SRS, 38).

Por eso, según el papa Francisco, se ha de trabajar desde la propia identidad, nacional o personal: "Se trabaja en lo pequeño, en lo cercano, pero con una perspectiva más amplia. Del mismo modo, una persona que

\footnotetext{
$8 \quad$ PP, 22; OA, 43; SRS, 32-33; CA, 35; Pablo VI, Discurso a la Organización mundial del trabajo (10.6.69); Juan Pablo II, Discurso al congreso de DSI (20.6.97) y Discurso a los dirigentes de sindicatos de trabajadores y grandes empresas (2.5.2000).
} 
conserva su peculiaridad personal y no esconde su identidad, cuando integra cordialmente una comunidad, no se anula sino que recibe siempre nuevos estímulos para su propio desarrollo. No es ni la esfera global que anula ni la parcialidad aislada que esteriliza" (EG, 235, 210).

\subsection{Fundamento del desarrollo}

El CDSI recuerda que el derecho al desarrollo se funda en los principios siguientes: unidad de origen y destino de la comunidad humana, igualdad entre todas las personas y de las comunidades basadas en la dignidad humana; destino universal de los bienes, desarrollo integral, centralidad de la persona humana, solidaridad.

El verdadero desarrollo ha de responder a la unidad de los siguientes niveles: el económico, el político y el de los valores, como expresión y fin de la unidad del género humano. Entre ellos deberá haber una unidad que responda a una escala de valores que, como señala J. L. Sampedro: "Frente a los niveles explicativos del desarrollo como resultado de la inversión, será preciso recordar que la decisión inversora tiene sus raíces no sólo en el nivel técnico-económico, sino también en el nivel político-social desde el cual se influye en la formación de esos precios o se orienta la inversión. Más aún, estas influencias, decididas en el seno de las instituciones insertas en ese segundo nivel, responden necesariamente a juicios, imágenes, preferencias o valores asentados en el tercer nivel" (Sampedro, 1987: 62).

Asimismo, el desarrollo verdadero se fundamenta y se mide por el parámetro interior. Aunque los bienes materiales son necesarios, sin embargo, ellos mismos han de estar en situación de ser respuesta a la vocación del hombre en Cristo. El desarrollo pone como centro al hombre, imagen de Dios, por ello el desarrollo no está tanto en el uso indiscriminado de los bienes cuanto "en la subordinación de la posesión, uso y dominio de los bienes a esa vocación humana" (SRS, 29).

De la misma manera, el auténtico desarrollo para ser pleno no sólo ha de respetar los derechos de los hombres sino además ha de ser consciente del valor de estos derechos. Este desarrollo será auténtico si se enmarca en el ámbito del respeto a la naturaleza, especialmente en la diferente valoración de los bienes y en su ordenación dentro del cosmos, con la consideración de la limitación de los mismos y la atención a la calidad de vida especialmente en zonas industrializadas.

Por todo esto, se puede afirmar que los pueblos tienen derecho a un desarrollo auténtico que sea integral, plasmado en el respeto y promoción de los derechos humanos, personales y sociales, económicos y políticos, el que tiene en cuenta las exigencias morales, culturales y espirituales. Se trata, por tanto, de un desarrollo humano integral (PP, 14; LS, cap. IV) y no 
sólo económico (Galindo García, 1988: 69-97; 2003a: 19-48). La razón es la siguiente: la cooperación va unida al respeto a la dignidad de la persona, de sus aspiraciones y de su creatividad.

\section{EVOLUCIÓN DE SU APLICACIÓN EN SRS, CV, EG Y LS'}

Conviene que nos situemos en una concepción humanista del desarrollo al reflexionar sobre el desarrollo sostenible auténtico, como culmen de la evolución del concepto de desarrollo y su aplicación en la Doctrina Social de la Iglesia desde la aparición de Populorum progressio. Esta concepción se encuentra especialmente, entre otros lugares, en las aportaciones de la Constitución Pastoral Gaudium et Spes, en la Encíclica Populorum progressio y en las encíclicas sociales publicadas por el Papa san Juan Pablo II (GS, 64; PP, 20; SRS, 34; CA, 37-38; EV, 10, 27, 43), en Caritas in veritate del papa Benedicto XVI y en algunas propuestas del papa Francisco en relación con la ecología y desarrollo en Laudato si, como aparece en el siguiente texto del Concilio: "La finalidad fundamental de esta producción no es el mero incremento de los productos, ni el beneficio, ni el poder, sino el servicio del hombre, del hombre integral, teniendo en cuenta sus necesidades materiales y sus exigencias intelectuales, morales espirituales y religiosas; de todo hombre, decimos, de todo grupo de hombres, sin distinción de raza o continente" (GS, 64).

Posteriormente, la encíclica Sollicitudo rei socialis insiste con el mismo interés en un momento histórico de crisis radical y universal cuando el hombre vive la tensión entre el deseo de cultivar un desarrollo humano pleno y ante la realidad de unas estructuras de pecado destructoras de este deseo. "Es, pues, necesario individuar las causas de orden moral que, en el plano de la conducta de los hombres, considerados como personas responsables, ponen un freno al desarrollo e impiden su realización plena. Igualmente, cuando se disponga de recursos científicos y técnicos, que mediante las necesarias y concretas decisiones políticas deben contribuir a encaminar finalmente los pueblos hacia un verdadero desarrollo, la superación de los obstáculos mayores sólo se obtendrá gracias a decisiones esencialmente morales, las cuales, para los creyentes y especialmente los cristianos, se inspirarán en los principios de la fe, con la ayuda de la gracia divina" (SRS, $35^{\circ}$ ).

Las propuestas morales al desarrollo nacen de la presentación del auténtico desarrollo. Este será descrito después de distinguir el progreso y el

9 Las fuentes de este texto son numerosas: PP, 44 veces; GS, 20 veces; RN, 2 veces; QA, 1 vez; MM, 3 veces; OA, 6 veces; LE, 3 veces; RH, 2 veces; Jornada por la Paz "Paz. con Dios creador. Paz con todo lo creado" 1990). 
desarrollo desde su fundamentación teológica. En este ámbito es preciso acercarnos al concepto de "sostenibilidad" respondiendo al objetivo de las organizaciones internacionales, entre las que se encuentra la Iglesia, de hacer propuestas ante el creciente mal uso del cosmos y sus repercusiones socioeconómicas entre los países del Norte y del Sur (Conferencia Episcopal Alemana "Por un futuro de solidaridad y de justicia", 28.2. 1997).

Por desarrollo sostenible, en cuanto camino del auténtico desarrollo, se entiende "aquel desarrollo que da respuesta a las necesidades del presente sin comprometer la capacidad de las generaciones futuras a satisfacer sus propias necesidades". Es decir, el desarrollo para ser sostenible debe tener como objetivo el satisfacer las necesidades esenciales de todas las personas que viven en este planeta según el principio de equidad y de justicia intergeneracional. Al mismo tiempo ha de desarrollarse sin destruir el ambiente natural.

Desde este horizonte se pueden reclamar tres dimensiones principales en este tipo de desarrollo. En primer lugar, la sostenibilidad ambiental exige conservar el capital natural respetando los recursos ambientales y los vínculos dados a la capacidad de regeneración de los sistemas ecológicos. En segundo lugar, la sostenibilidad económica exige integrar en el cálculo económico de una intervención además del capital y del trabajo, los sistemas naturales y el patrimonio común y artístico construido por la sociedad humana. Y, en tercer lugar, la sostenibilidad social exige el mejoramiento cualitativo de las condiciones de vida respeto y valoración del pluralismo cultural y de las tradiciones locales.

La sostenibilidad, por tanto, propone una aportación integral en la gestión de los procesos de una comunidad local, nacional e internacional buscando el equilibrio entre los diversos valores económicos, sociales y ambientales. Desde este ámbito, como he señalado en otro lugar (Galindo García, 2003b: 120ss), la reflexión debería desarrollarse proponiendo las tesis siguientes: la preocupación de la Iglesia por el desarrollo sostenible, el desarrollo como lugar teológico, la relación entre desarrollo y progreso y hacia el auténtico desarrollo con sus respectivas propuestas morales. En este sentido son tres las claves de tratamiento: la naturaleza como referencia, lo económico como medio transformador y la sociedad como sujeto.

El papa Francisco, como culmen del dinamismo de la reflexión sobre el desarrollo, resume su pensamiento sobre el desarrollo sostenible expuesto en Evangelii gaudium y en Laudato si, en su discurso dirigido a los asistentes al Congreso celebrado en Roma con Motivo de la celebración del 50 aniversario de Populorum progressio, con motivo de la puesta en marcha del Nuevo Consejo Pontificio para el Servicio del Desarrollo integral. En síntesis, insiste en los siguientes aspectos: 
$1^{\circ}$. En el 50 aniversario de la Encíclica Populorum progressio debemos recordar que fue el beato Pablo VI quien precisó en detalle en aquella encíclica el significado de "desarrollo integral" (PP, 21), y fue él quien propuso aquella fórmula sintética y afortunada: "de todo hombre y de todo el hombre" (PP, 14).

$2^{\circ}$. ¿Qué significa hoy y en el futuro próximo, desarrollo integral, es decir, el desarrollo de cada hombre y de todo el hombre? Siguiendo la estela de Pablo VI, tal vez en el verbo integrar - tan querido por el papa Francisco- se puede ver el horizonte del trabajo en favor del desarrollo humano. Veamos algunos aspectos.

a) Se trata de integrar los diferentes pueblos de la tierra. El deber de solidaridad nos obliga a buscar las maneras justas de reparto equitativo, para que no haya esa dramática desigualdad entre los que tienen mucho y los que nada tienen, entre el que descarta y el que es descartado. Sólo el camino de la integración entre los pueblos hace posible para la humanidad un futuro de paz y esperanza.

b) Es preciso ofrecer modelos viables de integración social. Todos tienen que dar una contribución a la sociedad en su conjunto, todos tienen una característica que puede servir para vivir juntos, nadie está excluido de aportar algo por el bien de todos. Esto es a la vez un derecho y un deber. Es el principio de subsidiariedad el que garantiza la necesidad de la aportación de todos, sea como individuos que como grupos, si queremos crear una convivencia humana abierta a todos.

c) Asimismo, dice el papa Francisco, es importante integrar en el desarrollo todos aquellos elementos que realmente hacen que sea así. Los diferentes sistemas: la economía, las finanzas, el trabajo, la cultura, la vida familiar, la religión son, cada uno en su especificidad, un momento irrenunciable de este crecimiento. Ninguno de ellos puede ser absoluto y ninguno de ellos puede ser excluido de un concepto de desarrollo humano integral, es decir, que tenga en cuenta que la vida humana es como una orquesta que suena bien, si los diferentes instrumentos se armonizan y siguen una partitura compartida por todos.

d) Por otra parte, no debe faltar la integración de la dimensión individual y la comunitaria. Es innegable que somos hijos de una cultura, al menos en el mundo occidental, que ha exaltado al individuo hasta convertirlo en una isla, como si se pudieran ser felices solos. Por otro lado, no faltan puntos de vista ideológicos y poderes políticos que han aplastado a la persona, la han masificado y privado de esa libertad sin la cual el hombre ya no se siente hombre. En esta masificación están también interesados poderes económicos que quieren explotar la globalización, en lugar de fomentar un mayor intercambio 
entre los hombres, simplemente para imponer un mercado global del que ellos mismos dictan las reglas y cosechan los beneficios.

e) Se trata, por último, de integrar cuerpo y alma. Ya Pablo VI escribió que el desarrollo no se reduce a un mero crecimiento económico (PP, 14); el desarrollo no consiste en tener cada vez más bienes a disposición para un bienestar puramente material. Integrar cuerpo y alma también significa que ninguna obra de desarrollo puede lograr su objetivo si no respeta el lugar donde Dios está presente para nosotros y habla a nuestros corazones.

f) Este concepto de desarrollo tiene una dimensión teológica (Galindo García, 2011a: 122ss.; 2012: 106ss.). "Dios se ha dado a conocer plenamente en Jesucristo: en Él, Dios y hombre no están ni divididos ni separados. Dios se hizo hombre para hacer de la vida humana, tanto personal como social, un camino concreto de salvación. Así, la manifestación de Dios en Cristo -incluyendo sus gestos de curación, de liberación y de reconciliación que hoy estamos llamados a proponer de nuevo a los muchos heridos al borde del camino- indica la senda y la manera del servicio que la Iglesia quiere ofrecer al mundo: a su luz, se puede entender lo que significa un desarrollo 'integral', que no sea contrario ni a Dios ni al hombre, porque asume la entera consistencia de ambos".

g) Pero también tiene una dimensión y amplitud antropológica propia. "En este sentido, el concepto de persona, nacido y madurado en el cristianismo, contribuye a perseguir un desarrollo plenamente humano. Porque persona siempre dice relación, no individualismo, afirma la inclusión y no la exclusión, la dignidad única e inviolable y no la explotación, la libertad y no la coacción”.

En este mismo horizonte dinámico, el papa Francisco se ha manifestado en Laudato si' al afirmar que "la realidad es más importante que la idea" (EG, 231-233). Una de las claves de la encíclica Laudato si', al igual que lo fue en Caritas in veritate (cap. VI), es la cuestión tecnológica. Para el papa Francisco no podemos resignarnos a las soluciones propuestas sólo por el paradigma tecnocrático. La razón está en que la tecnocracia nos impide ver la totalidad de la realidad de los problemas y las ambientes en que se encuentra la casa común y la humanidad.

Ante el enfrentamiento existente entre la idea y la realidad el papa Francisco propone el dialogo entre todos los agentes sociales, incluidos los representantes de las religiones, para fomentar el desarrollo sostenible: "la mayor parte de los habitantes del planeta se declaran creyentes, y esto 
debería provocar a las religiones a entrar en un diálogo entre ellas orientado al cuidado de la naturaleza, a la defensa de los pobres, a la construcción de redes de respeto y de fraternidad".

El principio de solidaridad, en atención a la realidad antes que a la idea, tiene aplicaciones prácticas entre las que el Papa enuncia la sostenibilidad de las ciudades, la electrificación rural y la concepción de nuevos proyectos. Y en el diálogo solidario entre los Estados es importante equilibrar las expectativas de progreso entre los países pobres y los ricos. Sobre la base de los debates entre países habría que asegurar el cumplimiento de los siguientes principios:

a) los países más avanzados tienen el deber moral de favorecer la transferencia hacia los países menos desarrollados el conocimiento actual necesario para utilizar eficientemente los recursos indispensables para su desarrollo.

b) el uso de las tecnologías energéticas tiene por parte de los países ricos la función de reducir la propia demanda con el de facilitar el acceso a las mismas por parte de los países pobres.

"Es indispensable prestar atención para estar cerca de nuevas formas de pobreza y fragilidad donde estamos llamados a reconocer a Cristo sufriente, aunque eso aparentemente no nos aporte beneficios tangibles e inmediatos: los sin techo, los toxicodependientes, los refugiados, los pueblos indígenas, los ancianos cada vez más solos y abandonados, etc. Los migrantes me plantean un desafío particular por ser Pastor de una Iglesia sin fronteras que se siente madre de todos. Por ello, exhorto a los países a una generosa apertura, que en lugar de temer la destrucción de la identidad local sea capaz de crear nuevas síntesis culturales. ¡Qué hermosas son las ciudades que superan la desconfianza enfermiza e integran a los diferentes, y que hacen de esa integración un nuevo factor de desarrollo! ¿Qué lindas son las ciudades que, aun en su diseño arquitectónico, están llenas de espacios que conectan, relacionan, favorecen el reconocimiento del otro!” (EG, 210) (Galindo García, 2008: 182ss.).

\section{ECOLOGÍA Y DESARROLLO}

Si bien es cierto que los primeros documentos pontificios, al respecto de cuestiones sociales, no tratan específicamente de problemas ambientales, en ellos podemos encontrar luces para discernir lo que podríamos llamar la concepción cristiana sobre la creación y el desarrollo humano. De todos modos, la reflexión de la DSI sobre desarrollo sostenible y ecología 
se va intensificando hasta el momento actual como puede verse en la enseñanza del papa Francisco.

León XIII en Rerum novarum (RN) recordando la Revelación (sobre todo el libro del génesis (Gn 1,28; ver también CDSI, 451) y valiéndose de Santo Tomás de Aquino (Suma contra Gentiles 1, II, c. II), nos enseña la relación entre el mundo creado y el hombre, sobre el derecho que el hombre tiene de usar de la naturaleza gozando del fruto del trabajo vinculado a la transformación de las cosas creadas y por ello de poseerla como propia, tomando en cuenta la necesidad de todos. Ello le da al hombre un derecho a la propiedad, pero no sólo individual sino colectiva, es decir la propiedad no deben ser tomada como propia sino como común (RN, 7ss.).

Pio XI, en la encíclica Quadragesimo anno (QA) reafirma esta enseñanza poniendo un énfasis mayor en la función social de la propiedad (QA, 45; ver también CDSI, 467).

Juan XXIII en la encíclica Mater et Magistra (MM) reafirmando las enseñanzas de RN y QA “... dentro del plan de Dios Creador, todos los bienes de la tierra están destinados, en primer lugar, al decoroso sustento de todos los hombres... y, al mismo tiempo, como ministros de la divina Providencia, al provecho de los demás" (MM, 119), pero advierte la necesidad de tomar en cuenta la necesidad de las generaciones futuras (MM, 79; ver también CDSI, 167).

Pablo VI en la encíclica Populorum progressio declara la necesidad de la industrialización para lograr el crecimiento económico y para el progreso humano, que es al mismo tiempo señal y factor de desarrollo (PP, 25; ver también CDSI, 180 \& 2; GS, 69). Pero en la Exhortación Apostólica Octogesima adveniens (OA), con relación a la movilización de las personas, urbanización que produce la industrialización y los nuevos estilos de vida, advierte que el hombre se puede estar volviendo esclavo de los bienes que el mismo produce (OA, 9; ver también CDSI, 458).

Propiamente la cuestión ambiental es tratada como un problema social con Pablo VI en OA cuando muestra como el hombre súbitamente se da cuenta que, fruto de la inconsiderada explotación de la naturaleza, la va destruyendo y junto va provocando también su propia destrucción (OA, 21).

Juan Pablo II en la encíclica Sollicitudo rei socialis, en la perspectiva del verdadero desarrollo humano nos dice que éste impone límites al uso de los recursos naturales, distinguiendo el "usar" del "abusar" como consecuencia de los nuevos estilos de vida consumistas.

Juan Pablo II da un carácter moral al uso de la creación, respecto a Dios y a los demás seres (cosmos) en una triple consideración: Tomar 
conciencia que no se puede usar impunemente de los seres creados animados e inanimados sin tomar en cuenta la naturaleza de cada uno en conexión con el sistema ordenado; la convicción de la limitación de los recursos naturales, algunos de los cuales no son renovables; y la consecuencia de ese tipo de desarrollo sobre la calidad de vida en zonas industriales teniendo en cuenta la contaminación que producen (SRS, 34).

En la encíclica Centesimus annus (CA), el Papa Juan Pablo II muestra como un gran desafío para el hombre actual con vista a las generaciones futuras es corregir los estilos de vida reflejados en el consumismo e incentivar nuevas concepciones de vida con las que se alcance la verdad, la belleza y el bien (CC, 36).

Más adelante nos dice que junto al consumismo y vinculado a él está la cuestión ecológica: "El hombre, impulsado por el deseo de tener y gozar, más que de ser y crecer, consume de manera excesiva y desordenada los recursos de la tierra y su misma vida" (CC, 37; ver también CDSI, 359).

Benedicto XVI en la encíclica Caritas in veritate (CV), plantea el tema del desarrollo muy unido a los deberes de la relación del hombre con el medio ambiente. Porque la naturaleza es un Don de Dios, su uso representa una responsabilidad para con los pobres, las generaciones futuras y toda la humanidad.

La naturaleza y el hombre en ella no es fruto del azar, si se pierde la noción de que es una creación y un Don de Dios como proyecto de amor y de verdad, se cae en el error de pensar que el medio ambiente es un tabú intocable, o por el contrario se abusa de él.

En este sentido es importante evitar una cierta "lucha de clases" del hombre con la naturaleza. Es contrario al verdadero desarrollo considerar la naturaleza como superior al hombre, como también es contrario proponer su completa tecnificación, porque el medio ambiente no sólo es "materia disponible" a nuestro gusto sino "obra admirable" de Dios (CV, 48; ver también CDSI, 451-487).

Dentro del problema medioambiental cumple un papel importante las cuestiones relacionadas a los cambios ambientales, los problemas energéticos (CDSI, 470) y las relaciones entre los países altamente tecnificados y los que están en vías de desarrollo.

Por una explotación desmedida de los recursos medioambientales, en países en vías de desarrollo y desarrollados se producen graves conflictos internos y externos, con muertes, destrucción y degradaciones; los países desarrollados son los que más consumen esos recursos, por tanto, deben 
urgentemente disminuir el propio gasto energético, mejorar la eficacia energética y buscar nuevas alternativas energéticas ${ }^{10}$.

La participación de todos (CDSI, 466), una autoridad mundial (CDSI, 469) y una normativa jurídica (CDSI, 468) sobre el recto uso de los recursos energéticos nos ayudarán a evitar conflictos entre países desarrollados y aquellos que están en vías de desarrollo e incluso conflictos internos entre los propios países, en unos por la falta de recursos energéticos para "la calidad de vida que llevan" y en otros porque su explotación conlleva depredación de su hábitat, entre otros. "Si quieres pazprotege la creación", lema de la Jornada mundial de la Paz 2010.

El uso indiscriminado del medio ambiente como "recurso" pone en peligro al medio ambiente como "casa" (CDSI, 461, 465), en especial de aquellos que habitan en ambientes biodiversos, comunidades indígenas, etc. (CDSI, 471).

Junto a esto, es necesario rescatar una conciencia sobre lo que Juan Pablo II llama "Ecología humana" (CA, 38) "11 ya que mentalidades y prácticas perversas que so pretexto de evitar desequilibrios demográficos y contra la voluntad, la libertad y la dignidad de la persona humana; intentan implementar formas de vida antinatalistas, con ello depredan al hombre no sólo en su derecho a existir sino en su propia esencia, ser dueño de sí, de la naturaleza que le ha sido dada por Dios y de su posibilidad de con ella trascender (CDSI, 464).

"No está demostrado siquiera que cualquier crecimiento demográfico sea incompatible con un desarrollo ordenado" (SRS, 25) ${ }^{12}$. Las sociedades tenidas como desarrolladas han logrado una conciencia ecológica grande, capaz de crear leyes que protegen el medio ambiente, generando en sus habitantes una cultura y una mentalidad capaces de incluso la inmolación a favor de la naturaleza; sin embargo y con la misma intensidad han promovido leyes, cultura y mentalidad antinatalistas que atentan contra la dignidad de la persona humana, esta es la mayor antinomia de la humanidad actual $(\mathrm{CV}, 51)$.

Hoy se dice mucho, se escribe, se legisla y se actúa para evitar desequilibrios ambientales, nunca estará de más, la Iglesia a través de su Doctrina Social incentiva a que el hombre actual tome conciencia y actúe en ese

10 Sobre esto ver "El Regreso de la Lucha de Clases". Disponible en http://sociedad.elpais.com/sociedad/2012/02/20/vidayartes/1329766843_742941.ht ml. Ver también Galindo García (2011b: 170ss.; 2010: 102ss.).

11 Ver también "El derecho a la vida y las políticas sociales en la Caritas in Veritate" de Stefano Fontana. Disponible en: www.vanthuanobservatory.org.

12 Ver también: “¿Qué tiene que ver el matrimonio y la fecundidad con la economía?”. Disponible en http://www.almudi.org/Noticias/tabid/474/ID/889/Que-tienen-quever-el-matrimonio-y-la-fecundidad-con-la-economia.aspx. 
sentido, desde este pequeño trabajo que no es otra cosa si un inicio de reflexión sobre el tema de fondo en la cuestión ambiental queremos hacer una última reflexión.

\section{PRINCIPIOS PARA UNA GRADUALIDAD Y ELABORACIÓN DEL PROPIO DESARROLLO}

Según lo dicho hasta ahora, constatamos que la dinámica del desarrollo necesita sustentarse en el fundamento de los principios de la Doctrina social de la Iglesia especialmente en los siguientes: Capacidad de iniciativa, Participación, Respeto a la cultura propia, Subsidiaridad-solidaridad, Globalización, respeto a la dignidad de la persona humana y búsqueda del Bien Común.

Principalmente se ha de procurar, bajo el principio de subsidiaridad, de la capacidad de creación del propio desarrollo bajo la libertad de iniciativa económica. "El principio de solidaridad, también en la lucha contra la pobreza, debe ir siempre acompañado oportunamente por el de subsidiaridad gracias al cual es posible estimular el espíritu de iniciativa, base fundamental de todo desarrollo socioeconómico, en los mismos países pobres" (CDSI, 449). Es preciso ayudar y convencer a los países pobres para que realicen ellos mismos su propio desarrollo y que adquieran progresivamente los medios para ello (PP, 55).

Puede constatarse en la sociedad moderna, quebrada por muchas desigualdades, que dentro del derecho de los hombres a participar activamente en la vida pública, sus posibilidades son mínimas. Ha de aceptarse el que los pueblos del tercer mundo tienen derecho a participar en las decisiones políticas de sus respectivos países ya que el hombre debe ser siempre el protagonista de su propio desarrollo (SRS, 30).

La evolución de la vida pública, no debe estar sólo en manos del Estado ni al servicio del mismo, sino al servicio del hombre (Maritain, 1983). Este derecho a construir el propio destino ha de ser pleno, es decir, ha de extenderse a lo económico y social, al mundo cultural y espiritual, aunque el camino de este desarrollo auténtico sea largo y complejo, dada la intrínseca fragilidad del hombre y la situación precaria originada por el carácter mutable de la vida humana (SRS, 38).

En el ser humano coexiste la tendencia a adquirir y acumular bienes poder y la tendencia a dar y compartir. Es la utopía cristiana quien ha de buscar unificarlas. Del interés propio no egoísta, si es un interés bien entendido, surge la creatividad, la innovación, el ahorro, la tenacidad y la asunción de riesgos. Todos ellos se impulsan cuando hay libertad en el país emprendedor. 
Aparece aquí una de las verdades más constantes afirmadas por el cristianismo: el principal recurso del hombre, junto con la tierra, es el mismo hombre: el hombre con su inteligencia, con su trabajo, con sus virtudes, es decir, su capacidad de conocimiento que ve la luz con el saber científico, su capacidad de organización solidaria.

Los resortes éticos ante el ejercicio de la propia capacidad han de replantear cuestiones como el uso legítimo de la propiedad de los medios de producción, el uso ideológico de la propiedad privada y los diferentes niveles de propiedad. En todo caso, el derecho a una participación en la posesión de los bienes de carácter personal ha de defender la libertad y dignidad de la persona, ha de asegurar un espacio vital para la familia y ha de asegurar las posibles acciones personales realizadas desde la libertad personal con iniciativa propia, responsabilidad y libertad en el uso de los bienes materiales, intelectuales y espirituales. Para ello, es necesario promover la libertad económica y política desde los ámbitos siguientes:

1) La libertad, como valor fundamental del hombre, debe estar unida a un interés inmediato, si se quiere demostrar que vale como fuerza aseguradora de una sociedad democrática. La libertad irá unida a la responsabilidad. Desde estas categorías humanas, sin perder la perspectiva social, ha de ser comprensible la licitud de promover el propio desarrollo.

2) Es de gran importancia la libre iniciativa tanto en la cultura del trabajo como de la empresa moderna en los países pobres: "hoy en día el factor decisivo es cada vez más el hombre mismo, es decir, su capacidad de conocimiento, que se pone de manifiesto mediante el saber científico, y su capacidad de organización solidaria, así como la de intuir y satisfacer las necesidades de los demás" (CA, 32).

3) Es preciso que se ayude a los países pobres a conseguir los conocimientos necesarios y a entrar en el círculo de las interrelaciones, a desarrollar actitudes para poder valorar mejor sus capacidades y recursos (CA, 34). "Todo esto se puede resumir afirmando una vez más que la libertad económica es solamente un elemento de la libertad humanas" (CA, 39); si el hombre es considerado más como un productor y consumidor que como sujeto que produce y consume pierde entonces su relación con la persona humana y termina por ser oprimido (CA, 37).

4) Podemos decir que para los sistemas de libre iniciativa y de mercado libre, también en los países pobres, el objetivo de la plena ocupación debe estar en el centro de todos los programas de política económica y laboral. Si este objetivo no se consigue queda comprometido la legitimidad ética del sistema social (CA, 38).

5) Se opone a la libre iniciativa la intervención del Estado asistencial causante de la crisis del "Estado del Bienestar" (CA, 39). "Al intervenir 
directamente y quitar responsabilidad a la sociedad, el Estado asistencial provoca la pérdida de energías humanas y el aumento exagerado de los aparatos públicos, dominados por lógicas burocráticas más que por la preocupación de servir a los usuarios, con enorme crecimiento de los gastos" (CA, 40). Los resortes éticos de la libre iniciativa son la respuesta idónea a la tensión surgida entre el poder de la mano invisible de la economía capitalista y la necesidad de la actuación de instancias intermedias en el conglomerado social que legitima el esfuerzo por acceder al propio desarrollo.

6) La libre iniciativa está cargada de sugerencias éticas: la primera es sin duda la profesionalidad. El país emprendedor llegará a conseguir unas realizaciones provechosas si responde a las intenciones, es decir, si los medios responden fielmente a los fines. El emprendedor ejercita un tipo de profesionalidad en la que asuma la relevancia de la innovación, de la iniciativa y de la decisión.

7) Pero dentro de un mundo tan complejo no es fácil ver con claridad todos los aspectos económicos, sociales y éticos. Por ello, el emprendedor ha de armarse de la discrecionalidad. Se trata de elegir aquello que es mejor sabiendo que estamos llamados a tomar conciencia de nuestra libertad en las decisiones diarias de nuestra existencia. Es en el ejercicio de esta libertad donde se pone de relieve el patrimonio ético de los países y las motivaciones más profundas de su existencia.

8) Asimismo, la utopía, en su dimensión ética, forma parte de la acción de la libre iniciativa. Todos amamos la utopía, pero no la violenta ni la ingenua. Necesitamos de una utopía que incluya el "realismo inteligente" que nos impulse adecuadamente a resolver los problemas de la complejidad económica y social. Se trata de orientar y de valorar la capacidad de decisión, innovación e iniciativa de los países pobres.

Por ello, según el papa Francisco, "al Estado compete el cuidado y la promoción del bien común de la sociedad. Sobre la base de los principios de subsidiariedad y solidaridad, y con un gran esfuerzo de diálogo político y creación de consensos, desempeña un papel fundamental, que no puede ser delegado, en la búsqueda del desarrollo integral de todos. Este papel, en las circunstancias actuales, exige una profunda humildad social" (EG, 240).

Esta cooperación de los pueblos y la ayuda al desarrollo de sus capacidades de iniciativa deben ser entendidas como un derecho inalienable de los países y pueblos más pobres al progreso cultural y económico. Para esto, ha de profundizarse en los valores democráticos y en la defensa de los derechos humanos reconocidos por todos como prueba de la disponibilidad. 
Desde estas claves existen algunas propuestas doctrinales y prácticas de la Iglesia en el ámbito del desarrollo. Las propuestas teóricas en el marco de influencia que la iglesia propone esta resumida en la carta que su eminencia Peter Ka Turkson, Presidente del Pontificio Consejo para la Justicia y la Paz presento en el Debate temático de alto nivel sobre la consecución de los objetivos de desarrollo sostenible Nueva York, 21 de abril de 2016.

En primer lugar, el papa valora positivamente los esfuerzos de naciones Unidas: "Cuando el Papa Francisco se dirigió a esta Asamblea el 25 de septiembre pasado, se refirió al Programa 2030 para el Desarrollo Sostenible como 'un importante signo de esperanza'. Esta esperanza, prosiguió, sólo se concretará si la Agenda se realiza de manera real, justa y efectiva, y aún más importante, si su marco es sostenible".

Asimismo, "La Santa Sede considera que la realización de la Agenda 2030 requiere más que financiación pública; también requiere financiación e inversión de acuerdo con los criterios basados en el valor de los inversores privados, como un complemento necesario de las finanzas públicas. De hecho, es necesario que actores no estatales, como grupos basados en la fe, lideren compromisos de múltiples partes interesadas en la actividad financiera ética para eliminar la desigualdad social y desarrollar una ambiciosa nueva agenda para cuidar mejor nuestro hogar común".

Por otra parte, "en su encíclica Laudato si", el Papa Francisco habla de "cuidado" y "cuidado". Porque, si uno se preocupa, uno está conectado, uno está involucrado y tocado. Cuidar es dejarse afectar por otro, tanto que el camino y las prioridades cambian. Con el cuidado, entonces, la línea dura entre el yo y el otro suaviza, borra, incluso desaparece. Así que cuando dejamos de lado algo precioso en el mundo, destruimos parte de nosotros mismos también, porque utilizamos estamos completamente conectados.

Por fin, "para realizar el Programa de Desarrollo 2030, estamos llamados a 'cuidar', incluso cuando se trata de finanzas. La actividad financiera éticamente irresponsable produce desigualdades sociales. Por el cuidado, estamos inspirados a la práctica de la financiación responsable y promover la inversión basada en el valor en la aplicación de la Agenda 2030".

De la misma manera, "como afirmó el Papa Pablo VI en 1967 en su encíclica 'Populorum progressio', el desarrollo es el nuevo nombre de la paz. La paz es la condición y el ambiente necesarios para cualquier desarrollo verdadero y duradero. En consecuencia, nuestro mundo conflictivo es probablemente el mayor reto para la realización del Programa de 2030. Las sociedades pacíficas y solidarias son más fundamentales que la disponibilidad de financiación y financiación”. 
Puede decirse que, como propuestas prácticas, con fundamento in re la Iglesia es la institución con más sub-instituciones trabajando en programas de desarrollo en todo el mundo. Multitud de instituciones religiosas, el programa misionero, y diversas ONGs de inspiración cristiana se esfuerza en promover el desarrollo integra en aquellos lugares y países en proceso de desarrollo.

La Iglesia propone a esta sociedad una antropología basada eminentemente en la sagrada escritura y en la reflexión occidental de índole racional y espiritual. El hombre como ser humano compuesto de memoria, inteligencia y voluntad caracterizado por el ejercicio de su libertad está lleno de posibilidades y de necesidades.

Su dimensión comunitaria le convierte en un ser relacional con otros de la misma especie con la naturaleza y con la trascendencia. Él se construye entre el pasado y el futuro en base al 'que' que le capacita y al 'para que' que le motiva. Se caracteriza por tanto por el tener al servicio del ser.

En resumen, siguiendo la ley de la gradualidad que el papa Francisco propone, el desarrollo de cada persona ha de ser gradual conforme a sus propias capacidades. Dígase lo mismo respecto a los pueblos de manera que el enemigo mayor del desarrollo gradual proviene del incumplimiento del principio de subsidiaridad que sucede cuando el primer mundo da las cosas hechas al tercer y cuarto mundo.

Es necesario enseñar a las gentes y a los pueblos a valerse por sí mismo sin perder la propia cultura e identidad. Por ello, el desarrollo de los pueblos necesita la ayuda de los países ricos para que ellos mismos fomenten su propio desarrollo.

\section{REFERENCIAS}

-Flecha Andrés, J. R. (1990). La teología del desarrollo. Estructuras de pecado. En Comentarios a la Sollicitudo rei socialis (pp. 21-53). Madrid: Ed. ASE.

-Galindo García, A. (1988). Dimensión moral del desarrollo. Corintios XIII, 47, 69-97.

-Galindo García, A. (2003a). La formación para la responsabilidad hacia lo creado y por un desarrollo sostenible. En Conferencia Episcopal Española, Pastoral del ambiente y ecología bumana (pp. 19-48). Madrid: EDICE.

-Galindo García, A. (2003b). Hacia el desarrollo auténtico y sostenible. Corintios XIII, 107-108, 95-130.

-Galindo García, A. (2005). La Cooperación internacional para el desarrollo. En J. T. Raga (coord.), Reflexiones para empresarios y directivos sobre el compendio de la Doctrina Social de la Iglesia (pp. 225-259). Madrid: Ed. ASE.

-Galindo García, A. (2008). Esperanza y solidaridad con las víctimas. En AA.VV., Salvados en esperanza. Comentarios a la encíclica de Benedicto XVI Spe Salvi (pp. 169-210). Salamanca: UPSA. 
-Galindo García, A. (2010). Exclusión social, crisis y Doctrina social de la Iglesia. Corintios XIII, 135, 103-131.

-Galindo García, A. (2011a). Principios y valores de la Doctrina Social de la Iglesia a la luz de Caritas in veritate. En Cátedra Cardenal Ernesto Ruffini, Desarrollo integral (pp. 50-75). Salamanca: UPSA.

-Galindo García, A. (2011b). Ecología en algunos encuentros internacionales. En J. R. Flecha (coord.), Ecología y ecoética (pp 75-96). Salamanca: UPSA.

-Galindo García, A. (2012). Horizonte antropologico de la DSI. En AA.VV. Razones para vivir y razones para esperar. Homenaje al prof. Dr. D. Jose Román Flecha Andrés (pp. 150-181). Salamanca: UPSA.

-Gamband, L. (2012). El mito del desarrollo sustentable, ciencia y naturaleza. Madrid: Ed. Kindle.

-Gray, J. (2011). Anatomía de Gray. Barcelona: Paidós.

-Guardini, R. (1958). El ocaso de la edad moderna. Madrid: Editorial Guadarrama.

-Longchamp, A. (2008). "Populorum progressio" hace cuarenta años. Un don de esperanza para la mundialización. Corintios XIII, 126, 157-169.

-Maritain, J. (1983). El hombre y el Estado. Madrid: Editorial Encuentro.

-Sampedro, J. L. (1987). El desarrollo, dimensión patológica de la cultura industrial. Madrid: Desarrollo.

-Segovia Bernabé, J. L. (2008). El derecho al desarrollo, dignidad y justicia. Corintios XIII, 126, 171-209.

-Zampetti, L. (1991). Estado y cultura en la Centesimus annus. En AA. VV., Tened en cuenta lo noble, lo justo, lo verdadero, Comentarios y texto de la encíclica "Centesimus annus" (pp. 150-165). Valencia: EDICEP.

Sumario: Introducción; 1. ¿Desarrollo o progreso?; 2. En el horizonte de la Agenda 2030; 3. Ante la propuesta de PP sobre desarrollo: derecho y fundamento; 3.1. Derecho al desarrollo; 3.2. Fundamento del desarrollo; 4. Evolución de su aplicación en SRS, CV, EG y LS'; 5. Ecología y desarrollo; 6. Principios para una gradualidad y elaboración del propio desarrollo; Referencias. 